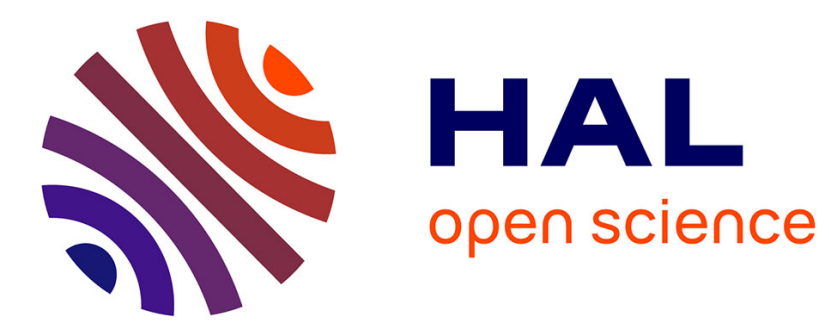

\title{
"Aryanisation" et spoliation des biens de la famille Jacob à Quimper
}

Patricia Sustrac

\section{To cite this version:}

Patricia Sustrac. "Aryanisation " et spoliation des biens de la famille Jacob à Quimper. Les Cahiers Max Jacob, 2021. hal-03467058

\section{HAL Id: hal-03467058 \\ https://hal.science/hal-03467058}

Submitted on 6 Dec 2021

HAL is a multi-disciplinary open access archive for the deposit and dissemination of scientific research documents, whether they are published or not. The documents may come from teaching and research institutions in France or abroad, or from public or private research centers.
L'archive ouverte pluridisciplinaire HAL, est destinée au dépôt et à la diffusion de documents scientifiques de niveau recherche, publiés ou non, émanant des établissements d'enseignement et de recherche français ou étrangers, des laboratoires publics ou privés. 


\title{
" ARYANISATION " ET SPOLIATION DES BIENS JACOB À QUIMPER
}

\author{
Patricia SUSTRAC* \\ " Je suis excessivement malheureux. \\ Les malheurs de ma famille me percent \\ le cour et la tête " \\ à Roger Toulouse, 24 janvier 1944.
}

Sous l'Occupation, les mesures de persécutions et d'exclusion de la population Nuive sont nombreuses. Elles découlent d'une législation concernant « le statut des Juifs » (octobre 1940 sq) et des lois économiques des 2 juin et 22 juillet 1941 anticipées par les décrets des autorités d'occupation (ordonnance du 27 sept. 1940). Les menaces et les persécutions pèsent autant sur la vie privée (interdiction de posséder un téléphone, une radio, une bicyclette ; limitation des déplacements...) que sur la vie professionnelle (« aryanisation » des entreprises).

La législation régissant les mesures de spoliation fait partie d'un arsenal de lois et de décrets qui ont méthodiquement détruit les acquis de la Révolution française ; jusqu'alors jamais le droit n'avait inscrit d'appartenances singulières. Il était en effet fondé sur la conception des droits de l'homme et construit sur le

* Patricia Sustrac, doctorante à l'université Toulouse Jean-Jaurès (sous la direction de Messieurs Patrick Marot et J.-Pierre Zubiate) a publié des articles critiques et biographiques, des bibliographies et édité plusieurs correspondances de Max Jacob. Elle est présidente de l'Association des Amis de Max Jacob depuis 2005, directrice de publication et secrétaire de rédaction des Cahiers Max Jacob. Elle partage avec Alexander Dickow la direction scientifique de la revue. Ils préparent la publication du Dictionnaire Max Jacob à paraître aux éditions Classiques Garnier et l'édition de la correspondance de Max Jacob à René Guy Cadou. 
postulat que tous bénéficiaient des mêmes droits ; tout ceci allait être bafoué au profit d'une législation antisémite durant ces quatre années d'occupation.

Cette législation est construite sur une triple volonté : repérer, exclure, exproprier. L'objectif est de « dégager» la société de l'influence des juifs et «purger » la vie économique du pays en « aryanisant » les propriétés « souillées » par leurs

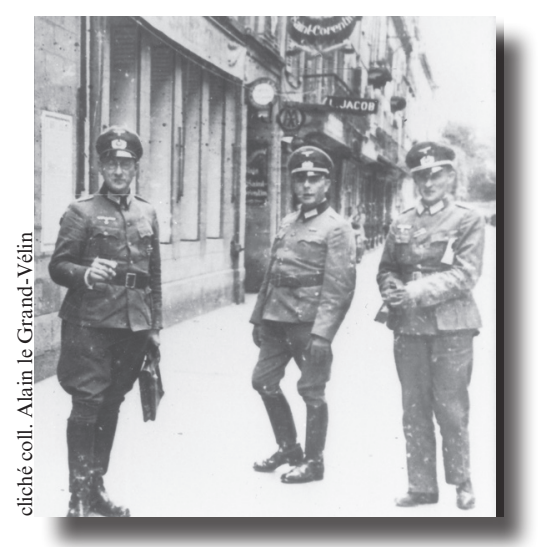

Officiers allemands devant le café de l'Épée ; en arrière- plan, l'enseigne du magasin des Jacob, juillet 1940 (détail).

propriétaires. L'idéologie nazie prétendait à une race « aryenne » supérieure a forgé ce néologisme « d'aryanisation » désignant par la suite l'ensemble des spoliations subies par les Juifs d'Europe ${ }^{1}$. Ce processus d'expropriation est un rouage essentiel de l'extermination; il interroge les rouages administratifs qui l'ont rendue possible ainsi que la dévolution des biens spoliés.

Vichy promulguera vingt-et-une lois ou décrets qui priveront les Juifs de leurs droits et les déposséderont de leurs biens. C'est une masse impressionnante de lois, de circulaires, de décrets qui se succèdent de 1940 à 1942 construisant « un droit antisémite » établissant " le Juif » comme une nouvelle catégorie juridique, véritable socle qui organise l'application des règles des discriminations spoliatrices. La spoliation est une dépossession légale cautionnée par un arsenal de dispositions règlementaires : «La légalité juridique habille les crimes d'État ${ }^{2} »$ rappelle l'historien Georges Bensoussan dans nombre de ses articles.

L'acquisition «d'un bien juif » est encouragée via d'intenses campagnes de presse. Le 23 juillet 1942, Le Matin publie, par exemple, un article au titre explicite : «Acquérir un immeuble juif est une excellente opération qui ne comporte aucun risque. » Trois points y sont développés : 1/ L'acquisition « d'un bien juif » est définitive. L'assertion s'appuie sur une logique dévoyée : «Les biens nationaux n'ont jamais été restitués, même pas au retour de la monarchie. » 2/ La loi du 21 juillet 1941 concerne les deux zones (alors que la France occupée est coupée en deux zones dont l'une est dite " libre. ») 3/ La procédure est simple et contrôlée : nomination d'un administrateur provisoire (AP) dont la mission est de contrôler la vente, d'évaluer le bien à sa juste valeur et de s'assurer que les acquéreurs soient " aryens », c'est-àdire non juifs. La clausule est sans ambiguïté : "L'acquéreur est assuré de jouir du revenu normal des capitaux par lui engagés. » On assistera à une curée « des biens juifs » qui deviennent une aubaine et l'occasion de s'accaparer le commerce ou l'appartement convoité de son voisin, parfois même 
de ses amis, sans scrupules. Preuve en est de l'entêtement de ces profiteurs à refuser de revenir à une légalité républicaine, les restitutions feront l'objet de nombreuses difficultés jusque dans les années $1950^{3}$.

Dans cet article, nous présentons les spoliations subies par Gaston et Delphine Jacob, frère et sœur aînés du poète Max Jacob. Nous ne dévoilerons ni les noms des acquéreurs de leurs biens, ni celui de l'AP chargé de leur administration malgré sa nomination au Journal officiel. Sous Vichy, les listes nominatives de toutes sortes étaient fréquentes. Il nous est impossible de souscrire à de pareils procédés admis dans les régimes autoritaires. Il est difficile aussi de vouer aux gémonies les descendants des acquéreurs et de l'AP. Nul n'est responsable de la faute de ses ancêtres. Nous ne localiserons pas non plus les biens acquis à l'exception de la maison du quai de l'Odet, de notoriété publique connue à Quimper sous le nom de "Villa Keristeir » ou de " Maison Jacob. » Nous bornons cet article aux aînés du poète et réservons pour une autre étude les spoliations subies par l'auteur et ses cadets, Jacques et Mirté Léa. La spoliation de la famille Gompel, cousine de celle du poète, a été étudiée par Philippe Verheyde (Les Mauvais comptes de Vichy, Perrin, 1999).

Concernant les vocables " bien juif ", " entreprise juive ", " aryanisation », nous prenons à notre compte l'analyse et la solution adoptée par Tal Bruttman $^{4}$ en adoptant l'usage systématique des guillemets afin d'établir une distanciation avec une logique que nous vomissons.

\section{LES JACOB : DES INDIVIDUS REPÉRÉS}

La loi du 22 juillet 1940 a ordonné la révision des naturalisations accordées par la loi de 1927. 15000 personnes sont concernées par cette destitution, 40\% sont des personnes de confession juive. Lazare Jacob, père du poète, a été naturalisé en août 1873 ; aucun des membres de sa famille ne perdra sa nationalité ; les fiches de greffe au camp de déportation de Drancy ou celui de Compiègne, les fiches de police, les fiches de convoi... tous ces documents précisent la nationalité française « d'origine » des internés et déportés Jacob.

Si la nationalité reste acquise, dans la foulée de la révision des naturalisations, la Commission de révision des changements de noms ${ }^{5}$ examinera, parmi d'autres, le cas des Jacob, le 14 mars 1944. Le jugement du 21 juin 1888 du tribunal de Tours autorisant la famille Alexandre à changer de nom pour Jacob est révoqué. La Commission décide un retour au patronyme d'origine. Le décret ne sera jamais promulgué, la Libération advenant plus vite que l'administration de Vichy ne le prévoyait. 
Les lois du 3 octobre 1940 fixe « le statut de Juif »; la loi du 2 juin 1941 ordonne le recensement et celle du 11 décembre $1942^{6}$ stipule l'apposition de la mention « Juif » sur les titres d'identité (§1). Les papiers personnels de Gaston et Delphine visés par les autorités n'ont pas été retrouvés - nous reviendrons sur ce point en évoquant le pillage de leur appartement et partant la disparition d'effets et de documents personnels et/ou privés (en l'espèce des documents ayant trait à leur famille et à Max Jacob en particulier.)

Dans le Finistère, cent trente-huit Juifs sont recensés en octobre 1940 ; trente d'entre eux sont localisés à Quimper ${ }^{7}$. Le recensement impose de déclarer sa confession; il devient dès lors un véritable piège aux conséquences tragiques : se déclarer Juif est une condamnation et ne pas se déclarer oblige à justifier d'une ascendance « aryenne » sur trois générations, ce que bien peu peuvent prouver. Et en tous cas pas les Jacob qui éluderont la réponse : «À Quimper, la confession israélite est revendiquée par vingt juifs dont cinq précisent que leurs épouses et leurs enfants sont catholiques [...] on compte également un catholique. Reste un groupe de cinq adultes, athées, agnostiques ou indifférents, pour lesquels ne figurent aucune mention. Parmi eux, Gaston et Delphine Jacob, frère et sœur de Max Jacob ${ }^{8}$.»

Le poète s'émeut de la situation de ses aînés; il se confie à Jean Colle le 4 octobre $1940^{9}:$ " Tu sais peut-être les nouvelles alarmantes publiées au sujet du sort des pauvres juifs (même convertis) dans les journaux et par la TSF. On doit se faire inscrire etc... et 500000 juifs étrangers sont internés dans des camps de concentration du Midi. Les professions libérales sont interdites aux autres ! Et il est probable que les ghettos seront bientôt rétablis. »

La loi du 22 juillet 1941 « relatives aux entreprises, biens et valeurs appartenant aux Juifs » cadre la spoliation économique, le rôle et la mission du Commissariat Général aux Questions juives (CGQJ) qui s'occupe de la " déjudaïsation » de l'économie ; celle du rôle central des AP (section 1); et du dispositif financier et prélèvements obligatoires sur le produit des ventes (§ 22). Enfin, la loi du 17 novembre règlemente l'accès des Juifs à la propriété foncière. L'ensemble de ces mesures qui affectent la vie personnelle et professionnelle de milliers de Juifs va concerner l'existence des aînés de Max Jacob et leur disparition ${ }^{10}$.

\section{CONTEXTE}

Le commerce créé par Samuel Alexandre dit Jacob circa 1858 à Quimper a toujours été une affaire familiale. La cession du bail s'est faite d'abord aux profits de Lazare et Maurice, père et oncle paternel du poète, puis Lazare rachète 
les parts de son frère. Prudence Jacob reprendra le commerce de son époux en 1917 à la mort de ce dernier. En 1937, Gaston et Delphine Jacob en deviennent propriétaires indivis après la mort de leur mère. Prudence avait en effet cédé ses parts à ses deux aînés le 26 juin 1920. Ils ont de suite formé le $1^{\text {er }}$ juillet une société en nom collectif au capital de $78615 \mathrm{f}$. « ayant pour objet l'achat, la vente et la mise en état de tous objets mobiliers ayant un caractère artistique d'antiquité $[\ldots]^{11}$. » La société est créée pour une durée de 20 ans soit jusqu'au

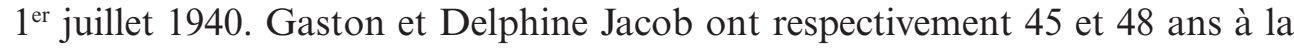
création de leur société, le calcul d'une retraite à 65 et 68 ans leur est sans aucun doute parue raisonnable. Célibataires sans enfants, la cession commerciale du bail n'a pas été envisagée.

Les Jacob n'ont jamais été propriétaires des murs du commerce ni de l'appartement situé au premier étage de l'immeuble du 8 rue du Parc où la famille a toujours résidé. Le magasin est loué aux Le Theuff, propriétaires du Café de l'Épée qui jouxte le magasin. Quant à l'appartement et aux dépendances en fond de cour et les ateliers, ils sont la propriété du chanoine Rossi.

La loi du 3 octobre $1940(\$ 4)$ interdit aux Juifs de posséder un commerce : fin octobre, le commerce est liquidé. Auparavant, il aura été identifié et désigné conformément à l'ordonnance allemande du 27 septembre 1940 (§ 4) qui impose le recensement « des entreprises juives » et son information en langue allemande et française avant le 31 octobre. Un panneau indiquant « Jude » sera apposé sur la vitrine du commerce. Une pancarte aura signalé quelques jours avant la fermeture du magasin : "Gros rabais - fin de saison » et une seconde : «Liquidation - Profitez des derniers jours ${ }^{12}$. » La location de l'appartement du $1^{\text {er }}$ étage étant corrélée à l'activité professionnelle, Gaston et Delphine quittent l'appartement familial. Jacob commente leur départ : «[...] Les deux vieux n’ont pas pu mieux faire que de liquider, de s'en aller vivre de ce qu'ils ont dans un appartement trouvé non sans peine au Pont Firmin, 34 rue Aristide Briand » (à Jean Colle, 4 novembre 1940).

Gaston et Delphine Jacob ne s'y sont pas établis " pour leur retraite » comme l'indique fâcheusement Anne Kimball dans l'édition des lettres à Julien Lanoë ${ }^{13}$ mais contraints par la persécution antisémite, en l'espèce l'interdiction de posséder un commerce. Leur nouveau domicile est la conséquence d'une dépossession et non une villégiature. Il n'est pas contestable que dans d'autres circonstances, ils eussent fini leur vie dans l'appartement familial.

On ignore le sort réservé à l'ameublement de la rue du Parc, la surface de la rue Aristide Briand ne pouvant accueillir le mobilier d'un vaste appartement bourgeoisement meublé14. Cependant, nous savons par Max Jacob qu'après l'arrestation de son frère, l'appartement sera " pillé, déménagé, sous scellés! avec tous les 
souvenirs $^{15}$ !! » (à Raphaël Arnal, 4 juillet 1943). À Jean Colle, le 8 janvier 1943, le poète avait déjà écrit l'acte sauvage et radical du pillage : « La haine a donc été jusqu'à dévaliser les derniers souvenirs que j'ai de ma famille ${ }^{16}$. » Il est donc impossible, comme nous le disions plus haut, de retrouver des documents personnels concernant les aînés de Max Jacob ni ceux que le poète auraient pu laisser chez ses aînés, à supposer qu'ils aient gardé ces documents dans leur déménagement.

Que s'est-il passé au 8 rue du Parc après le départ des Jacob ? Le 8 août 1942, un courrier rédigé en allemand de la Direction générale de la Police (service des Renseignements généraux) adressé au dirigeant du Commando extérieur informe de la situation professionnelle de Gaston Jacob et indique que le local commercial est occupé par le Secours national (AD 29). Le Recensement général des locaux d'habitation ou professionnel de $1947^{17}$ fait apparaître plusieurs occupants au $1^{\text {er }}$ étage : une lingère en journées, une commerçante - sans doute logées dans les pièces au-dessus des ateliers à l'équipement plutôt rudimentaire. Une famille de quatre personnes occupe l'appartement des Jacob. Le magasin, quant à lui, est toujours loué à l'organisme caritatif devenu L'Entraide française, relais régional d'un organisme de solidarité qui sera supprimé en 1949. Le Recensement ne donne pas en revanche la date d'entrée dans les lieux. Nul doute cependant que l'ensemble des locaux a été reloué rapidement.

\section{LES BIENS DE GASTON ET DELPHINE JACOB}

Le 30 décembre 1940, Max Jacob évoque la spoliation du commerce de ses aînés : «On prend aux juifs leurs maisons de commerce, si minces soient-elles et la majeure partie de leur fortune. C'est fait bien que les journaux n'en parlent pas $[\ldots] »$ (à Paul Petit, lettre inédite).

Ses aînés possèdent un patrimoine constitué de deux immeubles sis à Quimper et d'avoirs bancaires. Les biens fonciers proviennent d'une part d'un héritage et d'autre part d'une acquisition indivise ; les avoirs bancaires se répartissent en des portefeuilles d'actions et des liquidités personnelles et professionnelles déposées dans des comptes ouverts à l'agence quimpéroise du Crédit lyonnais. Ils possèdent en indivision leur commerce et les produits conséquemment obtenus après sa liquidation ainsi que $30 \%$ des parts de l'entreprise de bijouterie appartenant à leur beau-frère Lucien Lévy, mari de leur sœur cadette (cette entreprise sera placée sous contrôle d'un AP en 1941 et « aryanisée »). Ils perçoivent également des revenus de leurs biens loués (en 1936, un industriel marseillais, Gabriel Esun habite quai de l'Odet ; le rez-de-chaussée du bien sis dans le quartier Saint-Marc est loué à un commerçant). 


\section{LES PROPRIÉTÉS FONCIÈRES}

\section{Origine de PROPRIÉTÉ dU QUAI DE L'OdeT}

Gaston et Delphine ont hérité d'une maison construite par leur oncle et tante maternels, Raphaël et Delphine Jacob dite David, sœur cadette de Prudence, la mère du poète. Cette vaste et belle demeure a été érigée par les deux époux au cours de leur mariage selon les plans d'un pavillon russe de l'exposition universelle. Elle est bien connue de Max Jacob, il y est fréquemment reçu seul ou en famille. À Marcel Olin il raconte les moments passés dans le grand salon : « La vie se passe à écouter des histoires, à en faire des nouvelles, à faire des gaffes chez ma tante où je compromets mon héritage ${ }^{18}$ !»

À la mort de ses parents, la maison échoit pour moitié à leur fils Henri, et pour moitié en nue-propriété à Prudence Jacob. Henri décède le 21 juillet 1932 ; il a auparavant désigné Gaston et Delphine comme légataires universels. Le 9 février 1933, leur mère fait donation entre vifs de sa part « par preciput et hors part et avec dispense de rapport » avantageant ses deux aînés sur sa quotité disponible ${ }^{19}$.

À l'enterrement de son cousin germain, Max Jacob suffoque. Il est partagé entre la surprise - il n'a sans doute jamais été mis au courant des accords précités et la colère ; à son retour il se confie à Pierre Andreu le 25 juillet $1932^{20}$ :

Tu sais que j'ai passé quelques heures à Quimper pour l'enterrement d'un cousin mais tout a été si atroce que j'en suis parti comme la flèche quitte l'arc tendu. Tu vas certes sentir la profonde vérité de cette image là... Héritages! potage, brigandage, outrage, escamotage sauvage, tripotage ah! quittons quittons ces rivages anthropophages, soyons pauvres en notre ermitage plutôt que pauvre encore dans ces défenestrages. Ollé! Je suis volé (c'est laid), sans avoir la parole, chez des gens qui se disent honorable miroir ou famille. Je n'ai que mes amis et Dieu - un certain Dieu pitoyable au pécheur [...].

Max Jacob

homme soucieux et libre

Un texte rageur autobiographique titré « L'enterrement ${ }^{21}$ » conservé à la $\mathrm{BnF}$ décrit ce que l'auteur nomme « brigandages et escamotages » et finalement une captation d'héritage :

[Après l'enterrement] on rentra dans la famille. Quelle famille? On avait eu soin de ne prévenir aucun des vingt ou trente cousins pour qu'ils ne missent pas leur nez dans les affaires. Oh! quelle vengeance je tenais moi l'éternel sacrifié! l'éternel martyr. Moi qu'on évince de tout depuis 50 ans! [...] Je partis après les 
embrassades hypocrites habituelles et je passais dans le train la nuit à organiser un procès, à résumer mes arguments. Ceci fait, je décidais que ces gens m'avaient soigné un an dans ma dernière maladie et qu'un procès tuerait ma mère qui a 83 ans. Mais je crus pouvoir dans une lettre faire remarquer ma générosité et ma délicatesse. Mon frère me répondit une lettre triomphante et insolente [...]. Je démontrais qu'ils étaient passibles de correctionnelle pour captation d'héritage.

La pleine propriété de la maison en 1937 ne provoquera pas de réaction particulière de Max Jacob, sans doute par résignation. Nous ignorons les réactions des autres membres de la fratrie, le silence sans doute, l'acceptation sûrement. Cette maison ne leur appartient plus - nous y reviendrons.

\section{LA VENTE ${ }^{22}$}

L'acte de vente est dressé le 13 février 1941 au profit d'un entrepreneur quimpérois et de son épouse. La maison de pierre et de moellons couverte en ardoise est composée : sur une cave d'un rez-de-chaussée et d'un étage avec mansarde au-dessus. Agrémenté d'un terrain clos et d'un jardin, l'ensemble constitue une contenance de $500 \mathrm{~m}^{2}$. La maison est louée depuis novembre 1933 pour une durée de neuf années (soit jusqu'en 1942) moyennant un loyer annuel de $13000 \mathrm{f}$. La vente est réalisée selon un paiement échelonné : une rente viagère de $50000 \mathrm{f}$. laquelle sera réduite à $35000 \mathrm{f}$. au décès du premier mourant de l'un des vendeurs. L'AP sera nommé postérieurement à la vente - nous y reviendrons. Il sera chargé de valider la concordance entre la rente viagère et la valeur vénale du bien et de contrôler le dispositif financier. La vente a été validée conformément aux dispositions de l'article 2 de la loi du 16 novembre 1940 suivant autorisation préfectorale portant le $n^{\circ} 114$ en date du 22 janvier 1941. Les acquéreurs se déclarent aryens, catholiques et de nationalité française.

À la Libération, l'acquéreur aura versé aux crédits rentiers la somme de 285000 f. pour une propriété estimée à 528650 f. par l'AP dans son rapport d'expertise d'octobre 1942. Cependant, le nouvel acheteur ne profitera pas immédiatement de son bien. En effet, la maison est réquisitionnée par les troupes d'occupation ${ }^{23}$. Des travaux sont réalisés aux fins d'accueillir le Foyer du soldat/ Casino $^{24}$. L'indemnité de réquisition versée au nouveau propriétaire est égale au montant du dernier loyer connu, soit $13000 \mathrm{f}$. / annuel.

Du 3 au 8 août 1944, les combats font rage dans Quimper. La maison, située avantageusement sur l'Odet, devient un des lieux de repli des FFI ${ }^{25}$. Elle subit un certain nombre de dégâts. Il existe aux Archives départementales du Finistère un dossier de demande de réparations établi au nom de l'acquéreur (cote 1448 W 15). Néanmoins, le dossier vers lequel renvoit le 
numéro de la fiche est absent du bordereau des dossiers dommages de guerre $(214 \mathrm{~W})$. Nous ignorons donc si celui-ci a reçu une indemnité.

À la Libération, l'acquéreur est accusé d'avoir collaboré économiquement avec les Allemands ; une enquête de moralité est demandée par le Préfet de la Libération le 9 novembre 1944. Le rapport des Renseignements généraux conclura le 18 janvier 1945 à une conduite exemplaire durant l'Occupation : cache de personnes évadées, restriction de livraison de matériaux aux Allemands au bénéfice de clients français, don financier à la Résistance quimpéroise, participation aux combats de la libération de la ville... L'intéressé a par ailleurs fait valoir son témoignage par un courrier adressé au Commissaire des Renseignements généraux le 17 janvier. Il y détaille longuement ses activités, les raisons strictement professionnelles de la possession d'un ausweiss; il bat en brèche toute manœuvre d'enrichissement personnel illicite - l'acquisition des biens Jacob n'est pas évoquée. Preuve que le caractère légal de l'acquisition est bien enregistré dans les consciences. Lors de l'achat, l'acquéreur avait déclaré régler la rente viagère sur ses deniers personnels. Si en 1945, il admet avoir quelque fortune, elle est le « résultat d'une activité acharnée. » Il ne sera d'ailleurs pas poursuivi pour enrichissement illicite. Il donne les noms de personnalités quimpéroise de référence et joint des attestations de " bonne conduite " parmi lesquelles celle d'un de ses futurs locataires appartenant à sa hiérarchie professionnelle.

Reprenant possession de son bien, l'acquéreur effectuera des travaux de rénovation à fins de location peut-être avec les dommages de guerre. En 1947, le Recensement général des locaux à usage d'habitation ou professionnel décompte deux familles avec enfants locataires de la maison ${ }^{26}$. L'acquéreur vendra finalement son bien en 1952.

\section{L'IMMEUBLE DU QUARTIER SAINT-MARC}

Gaston et Delphine Jacob sont propriétaires indivis d'un immeuble acquis le 23 août 1926 pour la somme de 68000 f. Un bail commercial a été contracté le 25 septembre 1936 pour une durée de quatre années (loyer de 6000 f.). Les matrices des contributions foncières indiquent le nom et l'activité professionnelle du locataire : voyageur de commerce occupant en 1936. L'immeuble comprend une maison d'habitation, un jardin ayant grande porte sur rue, un poulailler et un puits sur une contenance de $255 \mathrm{~m}^{2}$. 


\section{LA VENTE}

L'immeuble est vendu par acte notarié du 26 février 1941. Elle est accordée selon un dispositif financier échelonné moyennant une rente annuelle viagère au profit des vendeurs et du "survivant d'eux sans réduction au décès du premier mourant, de quinze mille francs par $a^{27}$. " L'AP sera chargé de la vérification et de la validation de la vente par le CGQJ. Son rapport du 31 octobre 1942 récapitule les conditions de la transaction et désigne, suite au décès de Delphine en avril, Gaston comme attributaire unique de la rente. Après expertise du bien par un architecte, l'AP valide le montant concordant entre la rente à la valeur du bien (175000 f.). La vente a été validée conformément aux dispositions de l'article 2 de la loi du 16 novembre 1940 suivant autorisation préfectorale $n^{\circ} 89$ délivrée le 15 janvier 1941. L'acquéreur et son épouse déclarent être français et catholiques. Ils fournissent un certificat de baptême et un arbre généalogique.

L'acquéreur est entré immédiatement en possession de son bien. Notable établi solidement à Quimper, il jouit d'une grande respectabilité et concourt activement à la vie sociale et administrative de sa commune et de son département. Il est membre de nombreuses associations et d'organismes institutionnels qu'il préside souvent; il est aux commandes d'un organe d'information professionnel. Entrepreneur actif, il attire les jalousies : une enquête de moralité suite à une dénonciation de collaboration économique en 1942 sera rapidement levée par l'intéressé. À la Libération, il est assujetti au titre de l'Impôt de solidarité nationale (Archives Dépt. du Finistère, 75W116, n 2557, vol. 53, case 327). Impôt hors du commun, il porte sur les revenus et sur l'enrichissement sous l'Occupation ${ }^{28}$. La date d'arrêt est le 4 juin 1945, la date limite de déclaration février 1946. Le redressement se fonde sur les revenus perçus par l'administré.

Au dossier fiscal, le contribuable joint un préambule daté du 25 mars 1945 justifiant de ses activités durant l'Occupation, sa participation active à la Résistance et son arrestation par la Gestapo. Une déclaration détaillée de ses biens est annexée au dossier : liquidités personnelles, compte titres et comptes épargne, liste succincte de son mobilier, déclarations de ses véhicules dont il précise que l'achat est antérieur à 1939. Il détaille ses titres boursiers acquis entre 1941 et 1942 : essentiellement des actions et obligations de la Défense nationale. Il se déclare propriétaire de son logement, d'une résidence secondaire, d'un immeuble de rapport sis à Quimper et de plusieurs locaux professionnels, il loue également des entrepôts. Un court paragraphe concerne l'acquisition de l'immeuble des Jacob le 26 février 1941. L'acquéreur rappelle le dispositif du paiement de la 
rente et ses versements en 1941 et 1942 directement aux vendeurs (60 $000 \mathrm{f}$.) puis à partir de 1943 au liquidateur-syndic (l'AP). « Le sort de Monsieur Jacob n'ayant pas été élucidé et aucun héritier ne s'étant présenté, je n'ai pas pu verser de rente en 1944 et 1945. » $90000 \mathrm{f}$. auront donc été versés par l'acquéreur pour un bien estimé à $240000 \mathrm{f}$. en 1945 . Une note au tableau IV de la déclaration fiscale indique la déportation par les Allemands de Gaston Jacob et la nécessité d'une déclaration complémentaire si le décès était constaté - ce qui ne pourra être fait qu'en $1946^{29}$. En marge est noté : "S Surveillance. » Au titre du calcul de l'assiette de l'impôt, les montants versés au titre de la vente sont inscrits au passif, à cette date et par ce biais, l'acquéreur signale qu'il ne se considère pas comme propriétaire de l'immeuble. Aurait-il rendu son bien à Gaston Jacob ? À ses ayants-droit? Nous y reviendrons.

À la Libération, et de manière contradictoire à sa déclaration d'impôts, l'acquéreur va solliciter une indemnisation pour des dommages de guerre au titre de propriétaire. La posture montre l'ambivalence des consciences. Aux Archives départementales du Finistère, une fiche est conservée sous son nom sur laquelle est inscrite "Réglé par intendance de Vannes / Réparation immeuble. » En revanche, il manque à cette fiche un numéro de dossier et le dossier lui-même. Nous ignorons de quels dommages il peut s'agir. Le plan de Quimper sous l'Occupation allemande n'indique pas de bureaux ou d'accueil de troupes d'occupation dans ce quartier. Les dégâts sont-ils liés aux combats d'août 1944 ? À partir de 1946, les matrices des contributions foncières indiquent la pleine propriété de l'immeuble à l'acheteur et une activité commerciale de boucherie; la famille du commerçant occupe l'appartement situé au-dessus de la boutique et sous-loue à deux occupants.

\section{LES AVOIRS BANCAIRES ${ }^{30}$}

\section{COMPTES PERSONNELS}

Gaston Jacob possédait un compte de dépôts d'espèces et de titres n 2999 à l'agence de Quimper du Crédit lyonnais. Delphine Jacob possédait également un compte identique $\mathrm{n}^{\circ} 3000$ dans la même banque. Suite au blocage des comptes résultant de l'ordonnance allemande du 28 mai 1941 interdisant « la circulation des capitaux et le commerce des marchandises aux Juifs » et bloquant les comptes bancaires à hauteur de 15000 f. mensuels et de la loi de juillet 1941, l'agence quimpéroise déclare en janvier 1942 les comptes des Jacob au CGQJ. Les comptes sont classés sans AP, l'administrateur ayant été nommé en juin. 
Au 20 décembre 1941 les soldes étaient les suivants ${ }^{31}$ :

Gaston Jacob : solde de 6038,20 f. en espèces et $25037 \mathrm{f}$. en valeurs mobilières dont $24250 \mathrm{f}$. en rentes et obligations françaises ainsi que $787 \mathrm{f}$. en titres étrangers.

Delphine Jacob : solde de 5838,20 f. en espèces et de $25037 \mathrm{f}$. en valeurs mobilières dont $24250 \mathrm{f}$. en rentes et obligations françaises ainsi que $787 \mathrm{f}$. en titres étrangers.

En 1941, les Jacob déclarent leurs avoirs comme comptes de prélèvement uniques de subsistance. Gaston obtient une autorisation de prélèvement de 1600 f. mensuels, Delphine de 1700 f. mensuels ${ }^{32}$. En juillet 1942, une autorisation de prélèvement mensuel de $2500 \mathrm{f}$. a été demandée sur le compte de Gaston ainsi qu'une autorisation de prélèvement de $3000 \mathrm{f}$. sur le compte de Delphine décédée, sans doute pour payer les frais d'obsèques. Max Jacob présent aux funérailles de sa sœur ainée a pu constater le désarroi de son frère qu'il confie à André Salmon : "Il se perd dans des comptes sans fins et inutiles puisque tout son argent est bloqué à la banque et intangible » (30 avril 1942).

\section{Le COMPTE PROFESSIONNEL « D. ET G. JACOB ANTIQUitÉS »}

Un compte bancaire $n^{\circ} 2132$ a été ouvert au Crédit lyonnais. Il n'est pas déclaré par la banque comme un compte d'entreprise (identifié par des lettres et pas seulement des chiffres comme pour les comptes personnels). Il a plus vraisemblablement le statut d'un compte joint personnel. En juin 1942, l'agence quimpéroise déclare le compte au CGQJ sans AP et avec le solde suivant : espèces 1114,81 f.; titres : $6100 \mathrm{f}$. en obligations françaises.

Les valeurs sont des biens facilement mobilisables, ils ont permis de financer l'amende du milliard imposée aux «Juifs. »Prenant prétexte des attentats commis contre les troupes d'occupation, l'autorité allemande, par un avis du 14 décembre 1941, déclare que ces attentats commis par « des éléments à la solde des Anglo-saxons, des Juifs et des Bolcheviks » seront punis, non seulement par la déportation, l'exécution de " cent Juifs, communistes et anarchistes », mais aussi par l'imposition de cette amende d'un milliard de francs. L'administration des domaines chargée de la gestion des actions et portefeuilles versera 856 millions de francs à la Caisse des Dépôts et Consignations à cet effet. Les comptes des Jacob sont engloutis dans ce dispositif.

Les avoirs ont été transférés à la Caisse des Dépôts et Consignations, tombés en déshérence, ils ont été définitivement transférés à l'État ${ }^{33}$. 
Que peut-on en déduire ? Deux hypothèses s'offrent à nous. Les comptes ont pu être épuisés après les différents prélèvements opérés par l'AP. Cette hypothèse est fortement plausible au regard du découvert qu'il signale en juin 1943 pour un montant de 204 f. correspondant au montant des coûts d'expéditions des actes de vente des immeubles. La seconde hypothèse est qu'un dossier de succession ait été ouvert pour remise des avoirs à un notaire chargé de la succession. Toutefois, nous n'avons pas trouvé de dossier au nom de Gaston ou Delphine Jacob. La série déposée aux Archives Nationales est incomplète (et ces registres renvoient à des dossiers détruits ensuite après le délai de prescription de 30 ans). Aussi, en l'absence de toute indication nous ne pouvons infirmer ou confirmer l'hypothèse d'un versement de solde des comptes à une succession. Nous y reviendrons.

\section{L'ADMINISTRATEUR PROVISOIRE}

\section{UN RÔLE CAPITAL}

Les AP et les notaires ${ }^{34}$ sont les chevilles ouvrières de la spoliation. Vichy voudra rester décisionnaire et se substituer aux Allemands par l'intermédiaire du Service de contrôle des AP (SCAP) crée en décembre 1940 par le Ministère de la Production industrielle qui conduira le processus de « l'aryanisation. » $\mathrm{Ce}$ service passera rapidement sous l'autorité du CGQJ dont le rôle croissant peut masquer le contrôle de "l'aryanisation » par les forces d'occupation qui interviennent cependant à chaque étape du processus. Elle sont par exemple à l'origine des instructions pour les Commissaires-gérants des entreprises juives qui stipulent les missions des AP dont la «tâche [est] de supprimer définitivement l'influence juive dans l'économie française ${ }^{35}$. » Leur nomination est sous la ratification de la Militärbefehlshaber in Frankreich.

Dès la nomination d'un AP, le propriétaire est dessaisi de tous ses pouvoirs ; il perd la jouissance et la propriété de son bien ; il en est licencié quand il exerce une fonction dans son entreprise. La gestion revient à l'AP soit pour une administration du bien si celui-ci est nécessaire aux intérêts de clients français ou allemands soit pour préparer la vente. Le processus est fortement contrôlé par le CGQJ : les dossiers de Delphine et Gaston Jacob conservent plusieurs courriers adressés au Préfet pour validation des ventes, connaître la nature des versements reçus par « les juifs JACOB » et attester « l'aryanisation » des biens. Tous ces courriers ou notes internes du CGQJ s'expriment de cette manière : « La juive Jacob », « le juif Jacob », volonté manifeste de dépersonnalisation. 


\section{L'ADMINISTRATEUR PROVISOIRE DES BIENS JACOB}

Le 15 décembre 1943, la Caisse d'allocations familiales du Finistère ${ }^{36}$ demande confirmation de la liste des vingt-huit AP du département ${ }^{37}$. Proposé par le Préfet le 16 décembre 1941, l'administrateur provisoire des biens des Jacob est officiellement nommé par le CGQJ le 12 février suivant ; l'arrêté préfectoral est promulgué le 25 juin 1942, la parution au Journal officiel le 29 octobre 1942 (n² 259, p. 3588).

La nomination d'un AP est une procédure obligatoire ; elle valide officiellement les ventes qui resteraient sinon « nuls de plein droit» (loi du 22 juillet 1941 , § 4). La vente des biens ayant été faite avant la nomination d'un AP, les Jacob ont-ils agi avec précipitation ? Non. La spoliation débutant dès septembre 1940 en zone occupée (ordonnance allemande), ils vendent d'euxmêmes espérant tirer le meilleur parti des ventes, dans un contexte évidemment très menaçant ${ }^{38}$. Par ailleurs, Gaston et Delphine Jacob ne peuvent demeurer propriétaires comme nous l'avons souligné, la loi leur interdit la propriété foncière. Souhaitant sans doute se protéger, ils adoptent un système de cession via un dispositif de rente viagère supposé leur assurer un revenu régulier dans l'espérance de jours meilleurs. Ils encaissent d'ailleurs les premières échéances ; sécurité cependant illusoire puisque l'ordonnance allemande du 26 avril 1941 va interdire toute activité économique aux « Juifs » et les dépouiller de leurs biens. Dès lors, ils ne pourront plus disposer librement des produits des ventes ; leurs comptes bancaires seront bloqués (voir supra), la possibilité de leur verser des subsides sera possible en cas d'absolue nécessité avec l'aval de l'AP. Le dispositif financier des spoliations stipule que les produits des ventes sont versés à la Caisse des Dépôts et Consignations (compte $\mathrm{n}^{\circ}$ 501) au nom des spoliés. Conformément à la loi, un montant de $10 \%$ est prélevé après extinction du passif au bénéfice du CGQJ (compte ${ }^{\circ} 511$ ). La rémunération de l'AP est prélevée sur le produit des ventes.

Les ventes et le dispositif financier seront validés par les autorités allemandes et françaises. Cette homologation est décisive pour ratifier « l'élimination de l'influence juive effective et vérifier si le prix de vente est normal» (loi du 22 juillet 1941, § 14). Une expertise a d'ailleurs été demandée à un architecte près des Tribunaux pour évaluer le prix de vente des deux immeubles. Curieusement, le dispositif de la vente n'a pas été rejeté par le CGQJ et les autorités allemandes alors que la vente en viager est un dispositif peu courant. En effet, la loi stipule que les paiements échelonnés - s'ils sont admis -, doivent se solder six mois après la vente. Ce dispositif sera refusé, par exemple, pour l'échelonnement d'un délai de paiement sur quatre ans pour la vente du commerce de Jacques Jacob (AN-AJ/38/1915 dossier 736, avril 1941). 
Qui sont les acheteurs de ces biens et comment ont-ils eu connaissance de leur mise en vente puisque celle-ci n'a pas été soumise à publicité ? Pour l'immeuble du quartier Saint-Marc, il s'agit d'un voisin mitoyen - les Jacob devaient parfaitement connaître les besoins d'agrandissement de cet entrepreneur. Concernant la maison du quai de l'Odet, il s'agit d'un acheteur dont les activités professionnelles sont de première nécessité - sans aucun doute, les Jacob étaient de sa clientèle.

\section{RESTITUTION}

Dès avril 1941, le gouvernement de la France Libre communique sur les ondes de la BBC « qu'elle ne reconnaîtra aucun effet aux actes de confiscation $^{39} »$ : les torts seront réparés. À la Libération, le Service des restitutions est créé le 30 janvier 1945 en lieu et place du CGQJ. Les circulaires Terroine - directeur du Service d'octobre 1945 à 1946 - s'adressent en priorité aux spoliés dont les dossiers sont conservés dans ce sinistre service. Les envois recommandés avec accusé de réception à Delphine et Gaston Jacob sont restés lettres mortes, les enveloppes de retour ne figurent pas aux dossiers. Aucune recherche complémentaire ne semble avoir été entreprise par l'administration pour vérifier l'existence des intéressés, solliciter un acte de décès ou rechercher des héritiers.

Jacques Jacob et son épouse sont les seuls survivants de la fratrie. Robert Lévy (1909-1980), fils unique de Mirté Léa, la cadette, est interné en asile psychiatrique : il est dans l'incapacité de se constituer réclamant. Dès son hospitalisation en 1932, Robert Lévy a été pris en charge par le département de la Seine $^{40}$. Ce dispositif exclut d'office la question du paiement des frais d'hospitalisation lorsqu'il devint orphelin et jusqu'à son décès.

Jacques Jacob (1880-1960) a lui-même été victime d'une spoliation de son entreprise (AN-AJ38/1915 dossier 736). Son commerce lui sera restitué le 6 juillet 1945 (AD de Paris, 47W6). Sa vie pendant l'Occupation a été chaotique ; il a été arrêté, interné à Compiègne et a survécu tant bien que mal. En 1946, il entreprend des démarches pour obtenir les certificats de décès de ses aînés. Aucun dossier n'a été soumis auprès du Service de restitution des biens des victimes des lois et des mesures de spoliation.

Était-il d'ailleurs au courant du patrimoine de ses aînés ? On peut en douter. La maison du quai de l'Odet a " disparu » de la mémoire familiale en 1937 ; l'achat indivis de l'immeuble du quartier Saint-Marc était sûrement resté discret. Max Jacob a plusieurs fois souligné, au gré de sa correspondance, la pingrerie de « ses riches aînés » dont la situation patrimoniale est sans aucun doute restée 
opaque. Commerçant modeste, retraité discret, Jacques Jacob n'a jamais affiché un train de vie luxueux. Il est peu plausible qu'il ait sollicité le Service des restitutions pour recouvrer les avoirs de ses aînés.

En revanche, Jacques Jacob s'occupera activement de la restitution du corps de son frère aîné et de son transfert à Saint-Benoît-sur-Loire en 1949. Présent aux funérailles, il provoqua une très forte émotion tant la ressemblance avec son aîné était frappante.

\section{NOTES}

1 «Le substantif aryanisation et le verbe qui en découle n'existent pas dans la langue française [...]. [D']importation germanique - il semblerait que le terme provienne de la traduction de l'allemand Arisierung -, aryaniser signifie rendre aryen ou encore déjudaïser » (VERHEYDE Philippe, Les Mauvais comptes de Vichy : l'aryanisation des entreprises juives, Perrin, 1999, p. 18). Sur la langue inventée par l'idéologie nazie, voir KLEMPERER Victor, Lingua Tertii Imperii [la langue du troisième Empire], La langue du III ${ }^{e}$ Reich. Carnets d'un philologue, Agora Pocket, 2003.

2 BENSOUSSAN Georges, " éditorial », Revue d'histoire de la Shoah, Aryanisation : le vol légalisé, n 168 , nv ${ }^{1 \mathrm{le}}$ série, janvier-avril 2000, p. 4.

3 PROST Antoine, SKOUTELSKY Rémi, ÉTIENNE Sonia, Aryanisation économique et restitutions, La Documentation française, 2000.

4 «Évoquer des biens juifs relève d'une perversion du langage. L'usage aujourd'hui fait oublier le sens et la portée que recouvre [cette] expression : pour nombre d'antisémites, le fait que ces biens ont été détenus par des "mains juives" les a rendus juifs. Il ne s'agit pas tant de biens "juifs" que de "biens juifs", c'est-à-dire contaminés. La nuance peut paraître faible. Pourtant un exemple évocateur permet de comprendre au mieux la logique qui se trouve derrière ces mots : dans l'Allemagne nazie, les “animaux de compagnie juifs" seront tués, à l'égal de leurs propriétaires. » (BRUTMANN Tal, «Aryanisation » économique et spoliations en Isère (19401944), Grenoble : PUG, 2020, p. 7).

5 Arrêté du 10 février 1942, Journal officiel, p. 1936.

6 LOCHAK Danièle, Le Droit et les Juifs en France depuis la Révolution, Dalloz, 2009. Cet ouvrage recense toutes les lois et décrets allemands et français parus au Journal officiel pendant l'Occupation.

7 TOCZÉ Claude, LAMBERT Annie, Les Juifs en Bretagne ( $V^{e}-X X^{e}$ siècles), Rennes : PUR, coll. Mémoire commune, 2006, p. 166.

\& $\quad$ Ibid. p. 170

9 JACOB Max, Lettres à Jean Colle (1923-1943), transcription S. Lorant-Colle, annotations M. Dirou, Douarnenez : Mairie de Douarnenez, 1996, lettre du 4 octobre 1940, p. 34.

10 Julie Delphine est morte le 15 avril 1942 sans que l'on puisse déterminer s'il s'agit d'un suicide ou d'une mort naturelle. Quant à Gaston, arrêté le 16 juillet 1942, il est condamné à deux mois de prison (certificat ${ }^{\circ} 15315 / 15316,26$ sept. 1951, Archives du Ministère de la Défense, Service Historique de la Défense, Bureau des Archives des victimes des conflits européens). Il est arrêté 
à nouveau le 16 décembre (certificat $\mathrm{n}^{\circ} \mathrm{EC}$ 1389SAG $846 \mathrm{FN}, 29$ nov. 1950, idem). Dirigé vers Compiègne puis transféré à Drancy le 9 février 1943, Gaston est déporté à Auschwitz par le convoi $\mathrm{n}^{\circ} 47 \mathrm{du} 11$ février 1943. Les fiches individuelles de Gaston Jacob sont conservées au fichier individuel de la Préfecture de police et aux Archives nationales (cote F/9/5647/1), celles du fichier du camp de Drancy sous la cote F/9/5702/3. Aucun bien n'a été déposé au greffe à l'un ou l'autre camp, ce qui signifie que Gaston Jacob a été dépouillé des biens et objets personnels qu'il pouvait posséder antérieurement, cependant les registres d'écrous de la prison de Quimper manquent pour la période.

11 Acte dressé par Maître Le Berre, 26 juin 1920 : "Constitution de société », Le Finistère, 7 juillet 1920.

12 GRAAL Jean, Carnets 1938-1944, extraits juin à décembre 1940, Quimper : Centre Culturel Quimpérois, 2004, p. 50. Le catalogue de l'exposition La spoliation des Juifs, une politique d'État publie des documents graphiques (photos, des décrets et lois, fiches et dossiers administratifs ...) utiles à la compréhension du processus de dépossession (BRUTTMANN Tal (dir), Mémorial de la Shoah, mars 2013).

$13 \quad L A N O \ddot{E}, 21$ avril 1942, n. 1.

14 Le recensement de 1947 établit la liste des locaux du 34 rue Aristide Briand : ce sont des petites surfaces (mansardes, logements n'excédant pas trois pièces), AMQ, « Dossier 34 rue Aristide Briand. »

15 AAIII, p. 57.

16 Ibid. p. 43.

17 AMQ, Recensement général des locaux, 1947.

18 PJ, p. 34 [1911].

19 Par testament, Henri Jacob octroyait également une rente viagère annuelle à sa domestique Catherine Salaun de $800 \mathrm{f}$. qui sera également prélevée sur le produit de la vente (validation du CGQJ par lettre du 4 décembre 1943 pour effet par le Crédit lyonnais).

20 ANDREU Pierre, Max Jacob, éd. Wesmael-Charlier, coll. Les Convertis célèbres, 1962, p. 137.

21 Texte inédit, fds Gompel-Netter, BnF, NAF 28312/23.

22 Archives nationales, AJ38/4560, dossier 3893.

23 AMQ, cote $26 \mathrm{~J} 2$.

24 AMQ, « Dossier Maison Jacob », les travaux de rénovation sont conduits en nov. 1941, mémoire $n^{\circ} 20077,19 / 01 / 1942$, Service des réquisitions.

25 AD 29, fds Alain Le Grand, 208 J24.

26 AMQ, Recensement général des locaux 1947, cote 6 F18, 6 F7 et 6F22.

27 AN - AJ38/4560, dossier 392.

$28 \mathrm{AD} 29$, dossier en série $92 \mathrm{~W}$, service versant $\mathrm{n}^{\circ}$ 43. Je remercie Mme Émilie Goubin, responsable du secteur des archives contemporaines de son aide.

29 Gaston est né le 14 mai 1875 à Lorient et décédé le 16 février 1943 à Auschwitz. Le certificat de décès est dressé le 9 août 1946 conformément aux dispositions de l'ordonnance ${ }^{\circ} 452561$ du 30 octobre 1945, à la demande de Jacques Jacob, son frère cadet (Archives nationales AJ38/4560, pièce $\mathrm{n}^{\circ} 23.928 / 1$ sq.)

30 Je remercie Anne Brunterc'h, Responsable affaires générales du Crédit Agricole d'avoir facilité mes recherches.

$31 \quad$ AN - AJ 38/2782 et 2783.

$32 \quad$ AN - AJ38/773.

33 Voir Ciclade, site de recherche des comptes inactifs transférés à la Caisse des Dépôts et Consignations et supra n. 3 . 
34 LE COQ Vincent, POIROUX Anne-Sophie, Les Notaires sous l'Occupation (1940-1945), Acteurs de la spoliation des Juifs, Nouveau monde éd., 2015.

35 Commandement militaire en France, «Instruction pour les Commissaires-gérants d'entreprises juives », 12 octobre 1942, AN-AJ/ 38/4560.

36 AN-AJ/ 38/5438.

37 Ibid., ff. 1766-1778.

38 Cette démarche qualifiée faussement de «vente volontaire » sera d'ailleurs l'un des arguments des nouveaux acquéreurs refusant de rendre le bien spolié. C'est l'un des arguments évoqués par le nouveau propriétaire du commerce de Jacques Jacob à la Libération.

39 Appels de la France Libre diffusé sur les ondes de la BBC le 19 avril 1941 cité dans VERHEYDE Philippe, "L'aryanisation économique des grandes entreprises », Revue d'histoire de la Shoah, op. cit., p. 28.

40 AD 94, 4 X $414 s q$. 